\title{
Predictors of Reversion from Mild Cognitive Impairment to Normal Cognition
}

\author{
Seema Y. Pandya ${ }^{a, b}$ Laura H. Lacritz ${ }^{a}$ Myron F. Weinera \\ Martin Deschner ${ }^{\mathrm{a}}$ Fu L. Woon ${ }^{\mathrm{a}, \mathrm{c}}$ \\ a Department of Psychiatry, The University of Texas Southwestern Medical Center, Dallas, TX, \\ ${ }^{b}$ Department of Clinical and Health Psychology, University of Florida, Gainesville, FL, and \\ 'Seton Brain and Spine Institute, Department of Neurology, University of Texas at \\ Austin-Dell Medical School, Austin, TX, USA
}

\section{Keywords}

Mild cognitive impairment · Dementia - Reversion · Alzheimer disease · Cognition

\begin{abstract}
Background/Aims: Few studies have examined predictors of reversion from mild cognitive impairment (MCI) to normal cognition. We sought to identify baseline predictors of reversion, using the National Alzheimer's Coordinating Center Uniform Data Set, by comparing MCI individuals who reverted to normal cognition to those who progressed to dementia. Methods: Participants $(n=1,208)$ meeting MCI criteria were evaluated at the baseline visit and 3 subsequent annual visits. Clusters of baseline predictors of $\mathrm{MCI}$ reversion included demographic/ genetic data, global functioning, neuropsychological functioning, medical health/dementia risk score, and neuropsychiatric symptoms. Stepwise logistic regression models identified predictors of $\mathrm{MCI}$ reversion per cluster, which were then entered into a final comprehensive model to find overall predictor(s). Results: At 2 years, 175 (14\%) reverted to normal cognition, $612(51 \%)$ remained MCI, and 421 (35\%) progressed to dementia, with sustained diagnoses at 3 years. Significant variables associated with $\mathrm{MCI}$ reversion were younger age, being unmarried, absence of $A P O E$ \&4 allele, lower CDR-SOB score, and higher memory/language test scores. Conclusion: A relatively sizable proportion of $\mathrm{MCI}$ individuals reverted to normal cognition, which is associated with multiple factors previously noted. Findings may enhance MCI prognostic accuracy and increase precision of early intervention studies of dementia.
\end{abstract}


Pandya et al.: Predictors of Reversion from Mild Cognitive Impairment to Normal Cognition

\section{Introduction}

Mild cognitive impairment (MCI) does not always lead to dementia [1-5]. Incident rates of reversion to normal cognition among MCI individuals aged 65 years and older were noted to be up to $16 \%$ over 1 year in a clinic-based study [6] and $28-55 \%$ in population-based studies over a period of 2-12 years [2, 7-10]. Reasons for these varying rates of reversion are likely due to differences in MCI criteria, sample characteristics (e.g., inclusion of participants with transient medical and/or comorbid neuropsychiatric symptoms), length of follow-up, and/or test measurement error affecting MCI diagnostic accuracy [11].

Few studies have systematically focused on predictors of MCI reversion to normal cognition in comparison to the numerous studies on MCI progression to dementia. Of these studies, baseline predictors include higher neuropsychological test scores [9, 12], higher complex mental activity [10], and higher global functioning [6, 9, 13]. Additionally, younger age [6], being married [9], male gender [9], higher education level [13], nonamnestic MCI subtype [6], single domain $\mathrm{MCI}[6,10]$, absence of the apolipoprotein E (APOE) $\varepsilon 4$ allele [6, 9], and neither self-reported nor clinician-reported decline in memory [6] were predictors of reversion. Furthermore, larger hippocampal volume [10,12], fewer white matter hyperintensities [12], lower parahippocampal gyrus atrophy [13], and fewer Alzheimer disease (AD) biomarkers (i.e., higher cerebrospinal fluid plasma levels of amyloid-beta and lower cerebrospinal fluid plasma levels of tau proteins) [12] were predictive of reversion. These findings are not unexpected when considering that studies involving predictors of MCI progression have some findings in the opposite direction (e.g., education level higher for MCI reversion and lower for MCI progression). However, a few of the previous MCI reversion studies consisted of heterogeneous group compositions that likely affected the accuracy of their results and potentially undermined the precision in identifying predictive variables among MCI participants who fully revert to normal cognition [14]. Further, lack of a comprehensive model that includes a variety of other baseline variables (e.g., neuropsychiatric symptoms, medical health status) precludes understanding of MCI individuals with potential to revert to normal cognition.

The goal of the current study was to determine if baseline variables of MCI individuals help predict reversion to normal cognition by comparing those who reverted to normal cognition from MCI to those who progressed to a dementia over a 3-year follow-up period. To do so, we examined variables separated into 5 clusters related to baseline demographic/ genetic data, global functioning, neuropsychological functioning, medical health/dementia risk score, and neuropsychiatric symptoms. Results from our study may have several potential implications, including improved psychoeducation for patients and families on clinical outcomes of MCI, better informing healthcare providers on treatment management and clinical prognosis, and improved participant selection criteria in early intervention studies of dementia (e.g., increased study robustness).

\section{Methods}

\section{Setting and Participants}

The current longitudinal project incorporated a clinic-based population and utilized the National Alzheimer's Coordinating Center (NACC) Uniform Data Set (UDS) [15]. Data for participating MCI patients were submitted from each of the 30 Alzheimer's Disease Centers (ADCs) across the United States to the NACC between September 2005 and May 2013. Written informed consent was obtained from all participants and informants by each ADC, in compliance with their respective institute's committee on human research. For a more detailed description of the NACC UDS clinical evaluation form packet and its variables, see the article by Morris et al. [16]. The dementia risk score (developed from the population-based CAIDE 
Pandya et al.: Predictors of Reversion from Mild Cognitive Impairment to Normal Cognition

Table 1. Baseline demographic characteristics for MCI reversion and progression groups

\begin{tabular}{|c|c|c|c|c|c|c|}
\hline & $\begin{array}{l}\text { All subjects } \\
(n=596 ; 100 \%)\end{array}$ & $\begin{array}{l}\text { Reversion } \\
(n=175 ; 29.4 \%)\end{array}$ & $\begin{array}{l}\text { Progression } \\
(n=421 ; 70.6 \%)\end{array}$ & $t$ & $\chi^{2}$ & $p$ value \\
\hline Age, years & $74.07 \pm 8.95$ & $71.45 \pm 9.07$ & $75.16 \pm 8.68$ & -4.69 & & $<0.001$ \\
\hline Education, years ${ }^{\mathrm{a}}$ & $15.56 \pm 3.06$ & $15.75 \pm 3.05$ & $15.48 \pm 3.07$ & 0.96 & & 0.336 \\
\hline$A P O E \varepsilon 4$ allele $^{\mathrm{a}}$ & & & & & 25.88 & $<0.001$ \\
\hline 0 copies & $234(49.6)$ & $93(67.9)$ & $141(42.1)$ & & & \\
\hline$\geq 1$ copy & $238(50.4)$ & $44(32.1)$ & $194(57.9)$ & & & \\
\hline Gender & & & & & 7.20 & 0.009 \\
\hline Female & $300(50.3)$ & $103(58.9)$ & $197(46.8)$ & & & \\
\hline Male & $296(49.7)$ & $72(41.1)$ & $224(53.2)$ & & & \\
\hline Ethnicity & & & & & 3.70 & 0.076 \\
\hline Hispanic & $26(4.4)$ & $12(6.9)$ & $14(3.3)$ & & & \\
\hline Non-Hispanic & $570(95.6)$ & $163(93.1)$ & 407 (96.7) & & & \\
\hline Race $^{\mathrm{a}}$ & & & & & 17.00 & 0.005 \\
\hline White & $509(85.5)$ & $137(78.3)$ & $372(88.6)$ & & & \\
\hline Black/African American & $59(9.9)$ & $30(17.1)$ & $29(6.9)$ & & & \\
\hline Asian & $18(3.0)$ & $7(4.0)$ & $11(2.7)$ & & & \\
\hline Native Am./Alaskan & $1(0.2)$ & $0(0.0)$ & $1(0.2)$ & & & \\
\hline Native Hawaiian/Pac. Isl. & $1(0.2)$ & $0(0.0)$ & $1(0.2)$ & & & \\
\hline Other & $7(1.2)$ & $1(0.6)$ & $6(1.4)$ & & & \\
\hline Marital status $^{\mathrm{a}}$ & & & & & 9.35 & 0.003 \\
\hline Married & $422(70.9)$ & $108(62.1)$ & $314(74.6)$ & & & \\
\hline Unmarried & $173(29.1)$ & $66(37.9)$ & $107(25.4)$ & & & \\
\hline MCI subtype & & & & & 38.69 & $<0.001$ \\
\hline Amnestic & $504(84.6)$ & $123(70.3)$ & $381(90.5)$ & & & \\
\hline Nonamnestic & $92(15.4)$ & $52(29.7)$ & $40(9.5)$ & & & \\
\hline Source of cognitive complaint ${ }^{\mathrm{a}}$ & & & & & 117.70 & $<0.001$ \\
\hline Subject-report only & $48(9.5)$ & $30(26.8)$ & $18(4.6)$ & & & \\
\hline Informant-report only & $53(10.4)$ & $12(10.7)$ & $41(10.4)$ & & & \\
\hline Both subject and informant & $406(80.1)$ & $70(62.5)$ & $336(85.0)$ & & & \\
\hline
\end{tabular}

Values are means \pm SD or $n(\%)$. MCI, mild cognitive impairment; APOE $\varepsilon 4$, apolipoprotein E allele 4; MCI subtype amnestic = impairment in memory (single- or multi-domain). MCI subtype nonamnestic, non-memory impairment (single- or multidomain).

a Education data for 1 participant, race data for 1 participant, marital status data for 1 participant, APOE $\varepsilon 4$ data for 124 participants, and source of cognitive complaint data for 89 participants were not available.

study [17]), is a valid predictor of progression from normal cognition to dementia [18] that was not part of the NACC UDS. We calculated the risk score by using variables already available in the UDS. Study inclusion criteria involved participants who were diagnosed with MCI at the baseline visit using standard criteria [19], and had 3 annual follow-up visits. ADCs participating in the NACC used the following standard MCI criteria: (1) cognitive concern by the subject or informant; (2) clinician's impression or evidence of cognitive decline via objective testing; (3) abnormal cognition (neither normal nor demented), and (4) preserved functional activities via consensus or clinician diagnosis of MCI $[16,19]$. Exclusion criteria involved participants who had incomplete data (due to evaluations via telephone), were diagnosed with "Impaired/Not MCI" (i.e., an intermediate state between normal cognition and MCI) at 2nd and/or 3rd follow-up visits, or did not have the same diagnostic classification during their 2nd and 3rd follow-up visits (e.g., classified as "normal cognition" during the 2 nd follow-up visit but then as "MCI" during the 3rd follow-up visit). Included participants were then assigned to 1 of the 3 clinical outcome groups: (1) "MCI reversion" if they were classified as "normal cognition" at both the 2nd and 3rd follow-up visits; (2) "MCI progression" if they were diagnosed as "demented" at both the 2nd and 3rd follow-up visits; or (3) "MCI stability" if their diagnosis remained MCI at both the 2nd and 3rd follow-up visits; the above assignments were made regardless of the diagnostic status at the 1 st follow-up visit. 
Pandya et al.: Predictors of Reversion from Mild Cognitive Impairment to Normal Cognition

Table 2. Baseline data for clusters of global functioning, neuropsychological functioning, medical health/dementia risk, and neuropsychiatric symptoms for MCI reversion and progression groups

$\begin{array}{lll}\text { All subjects } & \begin{array}{l}\text { Reversion } \\ (n=175 ; 29.4 \%)\end{array} & \begin{array}{l}\text { Progression } \\ (n=421 ; 70.6 \%)\end{array}\end{array} \quad \begin{aligned} & \text { (n=596;100\%) } \\ & (n=17 \text { value }\end{aligned}$

Global assessments of functioning measures

\begin{tabular}{|c|c|c|c|c|c|}
\hline $\mathrm{CDR}^{-S_{O} B^{\mathrm{a}}}$ & $1.58 \pm 1.30$ & $0.62 \pm 0.61$ & $1.97 \pm 1.30$ & 17.20 & $<0.001$ \\
\hline $\mathrm{FAQ}^{\mathrm{a}}$ & $4.09 \pm 5.14$ & $1.05 \pm 2.73$ & $5.36 \pm 5.38$ & 12.91 & $<0.001$ \\
\hline MMSE $^{\mathrm{a}}$ & $26.90 \pm 2.45$ & $28.50 \pm 1.60$ & $26.24 \pm 2.44$ & -13.101 & $<0.001$ \\
\hline \multicolumn{6}{|l|}{ uropsychological measures ${ }^{b}$} \\
\hline LM story A Immediate Recall & $-0.97 \pm 1.27$ & $0.00 \pm 1.03$ & $-1.38 \pm 1.13$ & -13.60 & $<0.001$ \\
\hline LM story A Delayed Recall & $-1.13 \pm 1.36$ & $0.06 \pm 1.08$ & $-1.63 \pm 1.14$ & -16.25 & $<0.001$ \\
\hline Digit Span Forward & $-0.32 \pm 1.04$ & $-0.16 \pm 1.04$ & $-0.39 \pm 1.03$ & -2.40 & 0.017 \\
\hline Digit Span Backward & $-0.27 \pm 1.01$ & $-0.05 \pm 1.03$ & $-0.36 \pm 0.99$ & -3.44 & 0.001 \\
\hline Animal Fluency & $-0.77 \pm 0.95$ & $-0.37 \pm 0.97$ & $-0.93 \pm 0.89$ & -6.77 & $<0.001$ \\
\hline Vegetable Fluency & $-0.07 \pm 1.17$ & $0.60 \pm 1.16$ & $-0.35 \pm 1.06$ & -9.40 & $<0.001$ \\
\hline Trail Making Test part A & $-0.76 \pm 1.57$ & $-0.55 \pm 1.47$ & $-0.85 \pm 1.61$ & -2.07 & 0.039 \\
\hline Trail Making Rest part B & $-1.09 \pm 1.62$ & $-0.70 \pm 1.41$ & $-1.26 \pm 1.68$ & -4.02 & $<0.001$ \\
\hline Digit Symbol Test & $-0.51 \pm 1.03$ & $-0.09 \pm 0.93$ & $-0.69 \pm 1.02$ & -6.35 & $<0.001$ \\
\hline BNT & $-0.94 \pm 1.48$ & $-0.46 \pm 0.99$ & $-1.15 \pm 1.60$ & -6.15 & $<0.001$ \\
\hline \multicolumn{6}{|l|}{ edical health/dementia risk } \\
\hline CAIDE risk score & $6.75 \pm 1.89$ & $6.85 \pm 1.88$ & $6.71 \pm 1.90$ & -0.77 & 0.443 \\
\hline \multicolumn{6}{|c|}{ uropsychiatric symptoms (NPI-Q severity scores) } \\
\hline Anxiety & $1.41 \pm 0.55$ & $1.35 \pm 0.57$ & $1.43 \pm 0.55$ & 0.61 & 0.541 \\
\hline Apathy & $1.49 \pm 0.66$ & $1.46 \pm 0.66$ & $1.49 \pm 0.67$ & 0.14 & 0.888 \\
\hline Depression & $1.32 \pm 0.50$ & $1.17 \pm 0.38$ & $1.35 \pm 0.52$ & 2.20 & 0.079 \\
\hline
\end{tabular}

Values are mean \pm SD. CDR-SOB, clinical dementia rating sum-of-boxes (higher score signifies poorer functioning); FAQ, Functional Assessment Questionnaire (higher score signifies higher functioning); MMSE, Mini-Mental State Exam (higher score signifies higher functioning); LM, Wechsler Memory Scale-Revised Logical Memory (higher score signifies higher memory functioning); BNT, Boston Naming Test (higher score signifies higher language functioning); CAIDE, cardiovascular risk factors, aging and dementia (risk of dementia is $1.0 \%$ for score of $0-5,1.9 \%$ for score of $6-7,4.2 \%$ for score of $8-9,7.4 \%$ for score of 10-11, and 16.4\% for score of 12-15); NPI-Q, Neuropsychiatric Inventory Questionnaire. Range of NPI-Q symptom severity scores: 0-3 (i.e., " 0 " = none, " 1 " = mild, " 2 " = moderate, and " 3 " = severe).

${ }^{a}$ Raw score. ${ }^{b}$ Raw test scores were transformed to standard $z$-scores using demographically-adjusted norms (age, education, and gender) [21]. $z$-scores have a mean of 0 and SD of 1.

\section{Data Management and Statistical Analyses}

Descriptive statistics and comparisons between MCI reversion and progression groups at the univariate level were conducted for baseline data, using independent sample $t$ tests for continuous variables and $\chi^{2}$ tests for categorical variables. Mann-Whitney $U$ test as a nonparametric approach was used when variables were not normally distributed. All baseline variables for each cluster are located in Tables 1 and 2. Specifically, for the demographic/genetic data cluster, we examined age, gender, ethnicity, education level, marital status, $A P O E$ \& 4 status, and MCI subtype. Raw test scores from the neuropsychological test battery [20] were transformed to standard $z$-scores using demographically adjusted norms (age, education, and gender) [21]. To calculate CAIDE dementia risk scores, we recoded variables available in the NACC UDS packet to reflect the sublevel variables of the risk score (i.e., age, gender, education, systolic blood pressure, BMI, and total cholesterol). Each sublevel variable was assigned a weighted score and then summed to yield 1 CAIDE score per subject [17]. Anxiety, apathy, and depression variables from the Neuropsychiatric Inventory Questionnaire (NPI-Q) [22] in the NACC UDS packet were chosen based on their known associations with MCI progression to dementia [23, 24] and lack of data on their associations with MCI reversion to normal cognition.

At the multivariate level, a binary stepwise logistic regression model was used for each of the 5 clusters, with the dichotomized MCI outcome variable at the 3-year follow-up as "MCI reversion" versus "MCI progression" and selected demographic variables as covariates. To determine the demographic covariates, age, gender, ethnicity, and education were entered into a separate stepwise logistic regression model. All 
Pandya et al.: Predictors of Reversion from Mild Cognitive Impairment to Normal Cognition

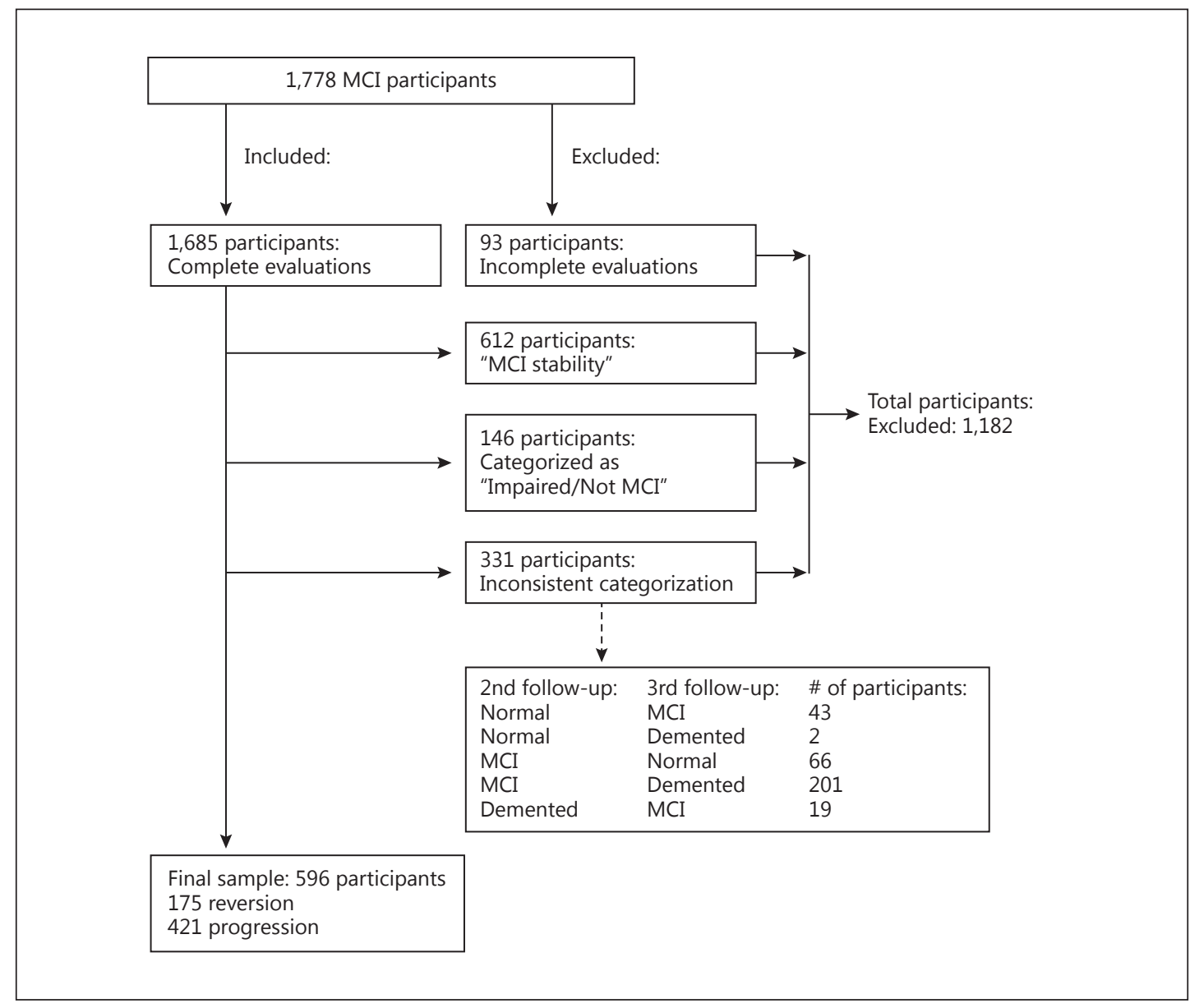

Fig. 1. Flowchart depicting the selection of the final study sample.

statistical analyses were performed using IBM SPSS Statistics version 21 and the 2 -sided $0.05 \alpha$ level was set as a criterion for statistical significance. Missing data were managed via pairwise exclusion.

A comprehensive model of prediction was used to determine the most significant predictors derived from the 5 clusters previously described. Significant results from each cluster were entered into a final binary stepwise logistic regression model with the outcome variable as "MCI reversion" versus "MCI progression". Furthermore, receiver operating characteristic (ROC) curves were used to determine cutoff scores for the continuous predictors and evaluate their prognostic value for MCI reversion. The area under the curve (AUC) classifications were based on established criteria [25].

\section{Results}

A study flowchart that describes the selection of the final sample is presented in Figure 1. A total of 1,778 participants were diagnosed with $\mathrm{MCI}$ at the baseline visit, from which 1,208 (68\%) participants were selected for this study after 1 of 2 rounds of inclusion and exclusion criteria. Of these 1,208 participants, 175 (14\%) were classified as MCI reversion, 612 (51\%) were classified as MCI stability, and 421 (35\%) were classified as MCI progression. Since the purpose of this study is to examine baseline predictors of MCI reversion compared to progression, the MCI stability group ( $n=612)$ was excluded in a second round of inclusion/exclusion criteria. Therefore, a total of 1,182 participants from the initial 1,778 MCI participants at the baseline 
Dementia

Cognitive Disorders

Table 3. Stepwise logistic regression analyses for baseline predictors of reversion

\begin{tabular}{l|l}
\hline \multicolumn{2}{l|}{ Dement Geriatr Cogn Disord 2017;43:2014-214 } \\
\hline DOI: $10.1159 / 000456070$ & $\begin{array}{l}\text { C 2017 S. Karger AG, Basel } \\
\text { www.karger.com/dem }\end{array}$ \\
\hline
\end{tabular}

Pandya et al.: Predictors of Reversion from Mild Cognitive Impairment to Normal Cognition

\begin{tabular}{|c|c|c|}
\hline Predictors & OR & $95 \% \mathrm{CI}$ \\
\hline \multicolumn{3}{|l|}{ Demographic/genetic data } \\
\hline Age & 0.93 & $0.91-0.96^{*}$ \\
\hline Education level & 1.03 & $0.95-1.11$ \\
\hline \multicolumn{3}{|l|}{ Gender } \\
\hline Female & 1.76 & $1.07-2.88^{*}$ \\
\hline Male (reference) & 1.00 & - \\
\hline \multicolumn{3}{|l|}{ Ethnicity } \\
\hline Hispanic & 2.28 & $0.72-7.25$ \\
\hline Non-Hispanic (reference) & 1.00 & - \\
\hline \multicolumn{3}{|l|}{ Marital status } \\
\hline Married & 0.48 & $0.28-0.83^{*}$ \\
\hline Unmarried (reference) & 1.00 & - \\
\hline \multicolumn{3}{|l|}{ MCI subtype } \\
\hline Nonamnestic & 3.43 & $1.94-6.07^{*}$ \\
\hline Amnestic (reference) & 1.00 & - \\
\hline \multicolumn{3}{|l|}{ APOE $\varepsilon 4$ allele } \\
\hline$\geq 1$ copy & 0.27 & $0.17-0.44^{*}$ \\
\hline 0 copies (reference) & 1.00 & - \\
\hline \multicolumn{3}{|c|}{ Global assessments of functioning } \\
\hline CDR-SOB $^{\mathrm{a}}$ & 0.31 & $0.20-0.46^{*}$ \\
\hline $\mathrm{FAQ}^{\mathrm{a}}$ & 0.84 & $0.76-0.94^{*}$ \\
\hline MMSE $^{a}$ & 1.52 & $1.32-1.76^{*}$ \\
\hline \multicolumn{3}{|l|}{ Neuropsychological measures ${ }^{\mathrm{b}}$} \\
\hline LM story A Immediate Recall & 1.04 & $0.70-1.55$ \\
\hline LM story A Delayed Recall & 2.81 & $1.93-4.09^{*}$ \\
\hline Digit Span Forward & 1.02 & $0.78-1.33$ \\
\hline Digit Span Backward & 0.90 & $0.67-1.22$ \\
\hline Animal Fluency & 1.03 & $0.74-1.42$ \\
\hline Vegetable Fluency & 1.35 & $1.04-1.75^{*}$ \\
\hline Trail Making Test part A & 0.83 & $0.68-1.01$ \\
\hline Trail Making Test part B & 1.10 & $0.90-1.35$ \\
\hline Digit Symbol Test & 1.53 & $1.10-2.13^{*}$ \\
\hline BNT & 1.51 & $1.19-1.91^{*}$ \\
\hline \multicolumn{3}{|l|}{ Medical health/dementia risk } \\
\hline CAIDE risk score & 1.04 & $0.94-1.15$ \\
\hline \multicolumn{3}{|c|}{ Neuropsychiatric symptoms (NPI-Q severity scores) } \\
\hline Anxiety & 0.67 & $0.45-0.99^{*}$ \\
\hline Apathy & 0.59 & $0.38-0.92^{*}$ \\
\hline Depression & 0.52 & $0.35-0.78^{*}$ \\
\hline
\end{tabular}

MCI, mild cognitive impairment; $A P O E \varepsilon 4$, apolipoprotein E allele 4; CDR-SOB, clinical dementia rating sum-of-boxes; FAQ, Functional Assessment Questionnaire; MMSE, Mini-Mental State Exam; LM, Wechsler Memory Scale-Revised Logical Memory; BNT, Boston Naming Test; CAIDE, cardiovascular risk factors, aging and dementia; NPI-Q, Neuropsychiatric Inventory Questionnaire. Number of cases per cluster of predictors included in the analysis: (a) demographic/genetic data = 470; (b) global assessments of functioning $=578$; neuropsychological measures $=515$; medical health $/$ dementia risk $=524$; neuropsychiatric symptoms $=565$.

${ }^{*} p<0.05$. ${ }^{\mathrm{a}}$ Raw score. ${ }^{\mathrm{b}}$ Raw test scores were transformed to standard $z$-scores using demographically-adjusted norms (age, education, and gender) [21]. 
Pandya et al.: Predictors of Reversion from Mild Cognitive Impairment to Norma Cognition

visit were excluded from the study (Fig. 1). A final sample of 596 participants was used for univariate and multivariate analyses. Among these final 596 MCI participants, 175 (29\%) were in the MCI reversion group and 421 (71\%) in the MCI progression group.

\section{Univariate Analyses}

Within the demographic/genetic data cluster, MCI reversion was significantly younger at baseline visit, had fewer females, had fewer copies of $A P O E \varepsilon 4$, and had more participants with nonamnestic MCI subtype than MCI progression (Table 1). Group comparisons for other cluster variables can be found in Table 2. Specifically, among the global functioning cluster, MCI reversion had significantly lower scores on CDR-SOB and FAQ and higher scores on MMSE than MCI progression at baseline. For neuropsychological functioning, MCI reversion had significantly higher baseline standard scores across all neuropsychological tests than MCI progression. Within the medical health/dementia risk score cluster and neuropsychiatric symptoms, no significant differences between the 2 groups were found.

\section{Multivariate Analyses: Binary Stepwise Logistic Regression Models}

Results from a separate stepwise logistic regression model for determining the demographic covariates showed that age $(\mathrm{OR}=0.96$; 95\% CI 0.94-0.98), gender $(\mathrm{OR}=1.67 ; 95 \%$ CI 1.15-2.42), and ethnicity (OR $=2.56$; 95\% CI 1.08-6.09) were significantly associated with $\mathrm{MCI}$ reversion and progression groups. Thus, they were selected as covariates in the stepwise logistic regression models for each of the clusters. The results of the multivariate analyses per cluster are presented in Table 3. Within the demographic/genetic data cluster, younger age, female gender, being unmarried, diagnosed as nonamnestic MCI, and absence of APOE $\varepsilon 4$ at baseline were significantly associated with MCI reversion. For global functioning, lower CDR-SOB and FAQ scores and higher MMSE scores at baseline were significantly associated with reversion. Of the neuropsychological variables, higher scores on Logical Memory Story A Delayed Recall, Vegetable Fluency, Digit Symbol, and Boston Naming Test (BNT) at baseline were significantly associated with MCI reversion (demographic covariates were not used because all neuropsychological test scores were already adjusted for gender, age, and education [21]). For the medical health/dementia risk score cluster, the CAIDE dementia risk score at baseline was not significantly associated with MCI reversion (demographic covariates were not used because age and gender contributed to the overall CAIDE risk score). Among the neuropsychiatric symptoms, lower symptom severity scores on anxiety, apathy, and depression at baseline were significantly associated with MCI reversion.

The overall comprehensive binary stepwise logistic regression model included the significant predictors from the above five clusters. Factors significantly associated with MCI reversion at baseline included younger age, being unmarried, absence of $A P O E \varepsilon 4$, lower CDR-SOB scores, and higher test scores on Logical Memory Story A Delayed Recall, Vegetable Fluency, and BNT (Table 4). Among these, ROC analysis for the continuous variables revealed AUCs of: a) 0.62 (95\% CI = 0.57-0.67) for age; b) 0.85 (95\% CI = 0.81-0.88) for CDR-SOB; c) $0.86(95 \% \mathrm{CI}=$ 0.83-0.89) for Logical Memory Story A Delayed Recall; d) 0.72 (95\% CI $=0.68-0.77$ ) for Vegetable Fluency; and e) $0.62(95 \% \mathrm{CI}=0.57-0.67)$ for BNT. Results further showed that Logical Memory Story A Delayed Recall standard $z$-score of -1.16 or better would accurately classify $M C I$ reversion from $M C I$ progression with $89 \%$ sensitivity and $73 \%$ specificity. Thus, a $z$ score of -1.16 is considered a valid cutoff value for this variable as a criterion for discriminating the MCI reversion group from the progression group. Cut off values were not established for CDR-SOB, because of its skewed distribution of scores (i.e., $0,0.5,1.0$, etc.), and Vegetable Fluency, due to a very low sensitivity (41\%) and high specificity (89\%). Furthermore, ROC curve analyses for age and BNT showed AUC values that were within the range of $0.60-0.70$, which suggests "poor" classification between the groups; therefore, we did not report those cutoff scores. 
Dementia

Cognitive Disorders

Table 4. Stepwise logistic regression analyses for comprehensive model of predictors for MCI reversion

\begin{tabular}{l|l}
\hline \multicolumn{2}{l}{ Dement Geriatr Cogn Disord 2017;43:2014-214 } \\
\hline DOI: 10.1159/000456070 & $\begin{array}{l}\text { C 2017 S. Karger AG, Basel } \\
\text { www.karger.com/dem }\end{array}$ \\
\hline
\end{tabular}

Pandya et al.: Predictors of Reversion from Mild Cognitive Impairment to Normal Cognition

\begin{tabular}{llc}
\hline Predictors & OR & $95 \% \mathrm{CI}$ \\
\hline Age & 0.91 & $0.87-0.96^{*}$ \\
Gender & & \\
$\quad$ Female & 0.35 & $0.11-1.10$ \\
$\quad$ Male (reference) & 1.00 & - \\
Marital status & & \\
$\quad$ Married & 0.32 & $0.14-0.76^{*}$ \\
$\quad$ Unmarried (reference) & 1.00 & - \\
MCI subtype & & \\
$\quad$ Nonamnestic & 1.32 & $0.50-3.48$ \\
$\quad$ Amnestic (reference) & 1.00 & - \\
APOE \&4 allele & & \\
$\quad \geq 1$ copy & 0.33 & $0.15-0.71^{*}$ \\
$\quad$ copies (reference) & 1.00 & - \\
CDR-SOB & 0.21 & $0.11-0.40^{*}$ \\
FAQ & 0.97 & $0.84-1.11$ \\
MMSE & 1.21 & $0.97-1.51$ \\
LM story A Delayed Recall & 2.39 & $1.68-3.39^{*}$ \\
Vegetable Fluency & 1.92 & $1.17-3.14^{*}$ \\
Digit Symbol Test & 1.36 & $0.89-2.08$ \\
BNT & 1.69 & $1.16-2.47^{*}$ \\
Anxiety & 0.90 & $0.41-1.98$ \\
Apathy & 0.84 & $0.35-2.02$ \\
Depression & 0.61 & $0.29-1.25$ \\
\hline
\end{tabular}

MCI, mild cognitive impairment; $A P O E \varepsilon 4$, apolipoprotein E allele 4; CDR-SOB, clinical dementia rating sum-of-boxes; FAQ, Functional Assessment Questionnaire; MMSE, Mini-Mental State Exam; LM, Wechsler Memory Scale-Revised Logical Memory; BNT, Boston Naming Test. Number of cases included in the analysis $=399{ }^{*} p<0.05$.

\section{Discussion}

This retrospective, clinic-based study, using data from past/current ADCs across the country, compared MCI reversion to MCI progression by examining a variety of variables to identify predictors of MCI reversion amongst individuals who reverted to normal cognition at 2 years and remained normal at 3 years. Specifically, 175 (14\%) of the 1,208 participants reverted from MCI to normal cognition at 2 years and remained normal at 3 years, 612 (51\%) remained MCI at 2 years and had a sustained diagnosis at 3 years, and 421 (35\%) progressed to dementia at 2 years and remained demented at 3 years. Our incident rate of reversion of $14 \%$ is higher than the range of reversion rates $(3-8 \%)$ found in other clinic-based MCI studies [12,13], yet is similar to the reversion rate of $16 \%$ noted by Koepsell and Monsell [6] over a 1-year follow-up. Possible reasons for this range of reversion rates (3-16\%) across studies include variable sample size, MCI criteria, follow-up periods, and classifications of study groups. Our progression rate of $35 \%$ is lower than a prior study by Lopez et al. [2] that followed MCI patients over 12 years and found a progression rate of $54 \%$ within 3 years. These incident progression rates are discrepant, likely because of the differing populations of interest and MCI criteria used at the baseline visit. Specifically, the present study is a clinicbased, case-control study that examined individuals diagnosed with $\mathrm{MCI}$ at baseline visit, while Lopez et al. [2] created an epidemiological study that examined cognitively normal individuals at the baseline visit and used the Cardiovascular Health Study-Cognition Study criteria for MCI. Nonetheless, it is likely that if the 421 participants in the MCI progression group of our study were followed for a longer period of time, the rate of progression would possibly 
Pandya et al.: Predictors of Reversion from Mild Cognitive Impairment to Normal Cognition

approximate to that of Lopez et al., in the context of the annual rate of progression being $10 \%$ as proposed in a meta-analysis study [3].

Variability in study design makes determining accurate rates of reversion and progression particularly difficult because there appears to be a lack of consistency in the literature as to how long after MCI diagnosis the trajectories of reversion or progression occurred. Additionally, the majority of available MCI reversion and progression studies did not report the number of $\mathrm{MCI}$ individuals who followed varying trajectories during the follow-up period (e.g., MCI - normal - MCI or MCI - normal - MCI - dementia). These "unstable" trajectories could affect the rates of reversion and progression and warrant further characterization in future studies. Thus far, 2 research groups $[2,9]$ have reported these "unstable" trajectories among their MCI patient samples, although without reporting the specific time points at which they occurred. Regardless, their findings are a starting point in understanding how the rates of MCI reversion and progression for those with "unstable" courses can differ from those with linear trajectories (i.e., MCI - normal or MCI - dementia).

We found a combination of 15 variables that significantly associated with reversion. of these, 7 remained significant in the final comprehensive model of prediction: younger age, being unmarried, absence of $A P O E \varepsilon 4$, lower CDR-SOB scores, and higher standard test scores on Logical Memory Story A Delayed Recall, Vegetable Fluency, and BNT, with Logical Memory Story A Delayed Recall being the sole predictor that could accurately classify MCI individuals into the reversion group versus the progression group, using a cutoff score of $z \geq-1.16$. This result is in agreement with findings by Gomar et al. [26] who used a comprehensive model to predict $\mathrm{MCI}$ progression to $\mathrm{AD}$ and found that tests of delayed verbal memory and left middle temporal lobe cortical thickness were the most significant predictors of MCI progression to $\mathrm{AD}$ over 2 years. Thus, delayed verbal memory is an important and accurate predictor for both MCI reversion and progression.

While the other 8 predictors (e.g., gender, MMSE score, etc.) were significant in the initial separate models for each cluster, the final model's inclusion of all significant predictors allowed for a multifactorial/dimensional framework that caused certain variables across clusters to correlate with each other. This, in turn, ultimately rendered those 8 variables nonsignificant because the variance they initially contributed was accounted for by the other 7 significant variables within the model. A comprehensive model of prediction is preferred versus an individually cluster-based one, since the present data suggest that MCI reversion, such as progression [26], is affected by multiple factors. Simply put, a multifactorial/dimensional approach is necessary in understanding MCI reversion.

Notably, our finding of being unmarried as a significant predictor of $\mathrm{MCI}$ reversion was discrepant from a previous MCI reversion study suggesting that being married was predictive [9]. Several factors could contribute to these discrepant findings. While social support and intellectual stimulation elicited by a spouse can help slow cognitive decline [27], stress due to the quality of the marital relationship could contribute to worsening cognition (e.g., caregiver burden/stress; patient believing caregiver is not providing sufficient care). Such stress would otherwise be nonexistent had the individual been unmarried. Notably, our study lacked data to assess the quality of the marital relationships. Another explanation for the discrepant findings could be differences in study methodology and/or sample characteristics. The research group estimated rates of reversion in an overwhelmingly older MCI sample (85\% aged 75 years and above; no mean age was provided), whereas we defined reversion with a sample group with a mean age of 71 years. Thus, the role of marital status in $\mathrm{MCI}$ reversion needs further validation.

Strengths of the current study include the use of a standardized protocol consisting of a comprehensive, annual evaluation that assessed a wide range of patient factors across past/ current ADCs in the country. Our study also included a large clinical sample with clearly 
Dementia

Cognitive Disorders \begin{tabular}{l|l}
\hline Dement Geriatr Cogn Disord 2017;43:2014-214 \\
\hline DOI: 10.1159/000456070 & $\begin{array}{l}\text { @ 2017 S. Karger AG, Basel } \\
\text { www.karger.com/dem }\end{array}$ \\
\hline
\end{tabular}

Pandya et al.: Predictors of Reversion from Mild Cognitive Impairment to Normal Cognition

defined/characterized MCI groups and a follow-up period longer than the majority of available MCI reversion studies. Such a study framework particularly differentiates our project from that of another reversion study [6], which focused on only a 1-year follow-up period and lacked clear group definition; that is, they combined cognitively normal individuals with an "Impaired/Not MCI group" (a separate, non-clearly defined diagnostic category) to produce a larger group classified as " $<\mathrm{MCI}$ ". Nonetheless, both studies shared the majority of the NACC UDS variables and noted a few similar findings of younger age, lower CDR-SOB scores, and lack of $A P O E \varepsilon 4$ as associated with MCI reversion.

Our study has some limitations. First, comparisons were made only between MCI reversion and MCI progression without examining MCI stability or Impaired/Not MCI, both of which may have distinct characteristics and/or predictors that require further exploration. Second, we did not explore imaging data because the NACC UDS standardized protocol did not include such information at the time of the data request. Third, it was unclear whether clinician judgment/classification systematically differs across the ADCs, although consensus diagnoses were 2-3 times more likely than single-clinician diagnoses to result in classification of MCI than normal cognition [28]. Finally, operationalization of the neuropsychological criteria (e.g., specific tests and impairment cutoffs) to help define MCI was unclear as neither the NACC UDS [16] nor Petersen [19] provide such definitions, which could also lead to inconsistent categorization of $\mathrm{MCI}$ and subsequent trajectories $[29,30]$.

In conclusion, a relatively large number of MCI patients do not progress to dementia and instead improve in their cognitive symptoms. Through a comprehensive model of analysis, we identified a number of factors significantly associated with MCI reversion. Future longitudinal studies with a larger scale framework, annual visits [31], and follow-up lengths greater than 3 years are needed, especially since $\mathrm{MCI}$ reversion can be a transitional or "unstable" state (e.g., MCI - normal - dementia; MCI - normal - MCI) and that such MCI patients may still be at risk for dementia at a later time [2,6,9]. This also supports the need for further MCI reversion studies to help differentiate the "false positive" MCI reverters (i.e., those with unstable courses who then progress to a dementia) from those who revert to normal cognition and remain cognitively normal for a number of years.

\section{Acknowledgments}

We would like to thank Matthew Clem for his assistance with data analysis in this project.

\section{Disclosure Statement}

There are no conflicts of interest to report. This work was supported by the Friends of the UT Southwestern Alzheimer's Disease Center. NACC database is funded by NIA/NIH Grant U01 AG016976. NACC data are contributed by the NIA funded ADCs: P30 AG019610 (PI Eric Reiman, MD), P30 AG013846 (PI Neil Kowall, MD), P50 AG008702 (PI Scott Small, MD), P50 AG025688 (PI Allan Levey, MD, PhD), P50 AG047266 (PI Todd Golde, MD, PhD), P30 AG010133 (PI Andrew Saykin, PsyD), P50 AG005146 (PI Marilyn Albert, PhD), P50 AG005134 (PI Bradley Hyman, MD, PhD), P50 AG016574 (PI Ronald Petersen, MD, PhD), P50 AG005138 (PI Mary Sano, PhD), P30 AG008051 (PI Steven Ferris, PhD), P30 AG013854 (PI M. Marsel Mesulam, MD), P30 AG008017 (PI Jeffrey Kaye, MD), P30 AG010161 (PI David Bennett, MD), P50 AG047366 (PI Victor Henderson, MD, MS), P30 AG010129 (PI Charles DeCarli, MD), P50 AG016573 (PI Frank LaFerla, PhD), P50 AG016570 (PI Marie-Francoise Chesselet, MD, PhD), P50 AG005131 (PI Douglas Galasko, MD), P50 AG023501 (PI Bruce Miller, MD), P30 AG035982 (PI Russell Swerdlow, MD), P30 AG028383 (PI Linda Van Eldik, PhD), P30 AG010124 (PI John Trojanowski, MD, PhD), P50 AG005133 (PI Oscar Lopez, MD), P50 AG005142 (PI Helena Chui, MD), P30 AG012300 (PI Roger Rosenberg, MD), P50 AG005136 (PI Thomas Montine, MD, PhD), P50 AG033514 (PI Sanjay Asthana, MD, FRCP), P50 AG005681 (PI John Morris, MD), and P50 AG047270 (PI Stephen Strittmatter, MD, PhD. 
Pandya et al.: Predictors of Reversion from Mild Cognitive Impairment to Normal Cognition

\section{References}

1 Ganguli M, Snitz BE, Saxton JA, Chang CC, Lee CW, Vander Bilt J, et al: Outcomes of mild cognitive impairment by definition: a population study. Arch Neurol 2011;68:761-776.

2 Lopez OL, Becker JT, Chang YF, Sweet RA, DeKosky ST, Gach MH, et al: Incidence of mild cognitive impairment in the Pittsburgh Cardiovascular Health Study-Cognition Study. Neurology 2012;79:1599-1606.

3 Mitchell AJ, Shiri-Feshki M: Rate of progression of mild cognitive impairment to dementia - meta-analysis of 41 robust inception cohort studies. Acta Psychiatr Scand 2009;119:252-265.

4 Petersen RC: Clinical practice. Mild cognitive impairment. N Eng J Med 2011;364:2227-2234.

5 Sachdev PS, Blacker D, Blazer DG, Ganguli M, Jeste DV, Paulsen JS, et al: Classifying neurocognitive disorders: the DSM-5 approach. Nat Rev Neurol 2014;10:634-642.

6 Koepsell TD, Monsell SE: Reversion from mild cognitive impairment to normal or near-normal cognition: risk factors and prognosis. Neurology 2012;79:1591-1598.

7 Ganguli M, Dodge HH, Shen C, DeKosky ST: Mild cognitive impairment, amnestic type: an epidemiologic study. Neurology 2004;63:115-121.

8 Han JW, Kim TH, Lee SB, Park JH, Lee JJ, Huh Y, et al: Predictive validity and diagnostic stability of mild cognitive impairment subtypes. Alzheimers Dement 2012;8:553-559.

9 Roberts RO, Knopman DS, Mielke MM, Cha RH, Pankratz VS, Christianson TJH, et al: Higher risk of progression to dementia in mild cognitive impairment cases who revert to normal. Neurology 2014;82:317-325.

10 Sachdev PS, Lipnicki DM, Crawford J, Reppermund S, Kochan NA, Trollor JN, et al: Factors predicting reversion from mild cognitive impairment to normal cognitive functioning: a population-based study. PloS One 2013; 8:e59649.

11 Lezak MD, Howieson DB, Bigler ED, Tranel D: Neuropsychological Assessment, ed 5. New York, Oxford University Press, 2012.

12 Park MH, Han C: Is there an MCI reversion to cognitively normal? Analysis of Alzheimer's disease biomarkers profiles. In Psychogeriatr 2015;27:429-437.

13 Tokuchi R, Hishikawa N, Kurata T, Sato K, Kono S, Yamashita T, et al: Clinical and demographic predictors of mild cognitive impairment for converting to Alzheimer's disease and reverting to normal cognition. J Neurol Sci 2014;346:288-292.

14 Pandya SY, Clem MA, Silva LM, Woon FL: Does mild cognitive impairment always lead to dementia? A review. J Neurol Sci 2016;369:57-62.

15 Beekly DL, Ramos EM, Lee WW, Deitrich WD, Jacka ME, Wu J, et al: The National Alzheimer's Coordinating Center (NACC) database: the Uniform Data Set. Alzheimer Dis Assoc Disord 2007;21:249-258.

16 Morris JC, Weintraub S, Chui HC, Cummings J, Decarli C, Ferris S, et al: The Uniform Data Set (UDS): clinical and cognitive variables and descriptive data from Alzheimer Disease Centers. Alzheimer Dis Assoc Disord 2006; 20:210-216.

17 Kivipelto M, Ngandu T, Laatikainen T, Winblad B, Soininen H, Tuomilehto J: Risk score for the prediction of dementia risk in 20 years among middle aged people: a longitudinal, population-based study. Lancet Neurol 2006;5:735-741.

18 Exalto LG, Quesenberry CP, Barnes D, Kivipelto M, Biessels GJ, Whitmer RA: Midlife risk score for the prediction of dementia four decades later. Alzheimers Dement 2014;10:562-570.

19 Petersen RC: Mild cognitive impairment as a diagnostic entity. J Int Med 2004;256:183-194.

20 Weintraub S, Salmon D, Mercaldo N, Ferris S, Graff-Radford NR, Chui H, et al: The Alzheimer's Disease Centers' Uniform Data Set (UDS): the neuropsychological test battery. Alzheimer Dis Assoc Dis 2009;23:91.

21 Shirk SD, Mitchell MB, Shaughnessy LW, Sherman JC, Locascio JJ, Weintraub S, et al: A web-based normative calculator for the uniform data set (UDS) neuropsychological test battery. Alzheimers Res Ther 2011;3:32.

22 Kaufer DI, Cummings JL, Ketchel P, Smith V, MacMillan A, Shelley T, et al: Validation of the NPI-Q, a brief clinical form of the Neuropsychiatric Inventory. J Neuropsychiatry Clin Neurosci 2000;12:233-239.

23 Jorm AF: History of depression as a risk factor for dementia: an updated review. Aust N Z J Psychiatry 2001; 35:776-781.

24 Somme J, Fernandez-Martinez M, Molano A, Zarranz JJ: Neuropsychiatric symptoms in amnestic mild cognitive impairment: increased risk and faster progression to dementia. Current Alzheimer Res 2013;10:86-94.

25 Greiner M, Pfeiffer D, Smith RD: Principles and practical application of the receiver-operating characteristic analysis for diagnostic tests. Prev Vet Med 2000;45:23-41.

26 Gomar JJ, Bobes-Bascaran MT, Conejero-Goldberg C, Davies P, Goldberg TE; Alzheimer's Disease Neuroimaging Initiative: Utility of combinations of biomarkers, cognitive markers, and risk factors to predict conversion from mild cognitive impairment to Alzheimer disease in patients in the Alzheimer's disease neuroimaging initiative. Arch Gen Psychiatry 2011;68:961-969.

27 Hakansson K, Rovio S, Helkala EL, Vilska AR, Winblad B, Soininen H, et al: Association between mid-life marital status and cognitive function in later life: population based cohort study. BMJ 2009;339:b2462.

28 Steenland K, Macneil J, Bartell S, Lah J: Analyses of diagnostic patterns at 30 Alzheimer's disease centers in the US. Neuroepidemiology 2010;35:19-27.

29 Loewenstein DA, Acevedo A, Small BJ, Agron J, Crocco E, Duara R: Stability of different subtypes of mild cognitive impairment among the elderly over a 2- to 3-year follow-up period. Dement Geriatr Cogn Disord 2009;27:418-423.

30 Teng E, Tingus KD, Lu PH, Cummings JL: Persistence of neuropsychological testing deficits in mild cognitive impairment. Dement Geriatr Cogn Disord 2009;28:168-178.

31 Weiner MW, Veitch DP, Aisen PS, Beckett LA, Cairns NJ, Green RC, et al: The Alzheimer's Disease Neuroimaging Initiative: a review of papers published since its inception. Alzheimers Dement 2012;8(suppl 1):S1-S68. 